\title{
An $x$-ray microscopic study of the normal root of neck arteries in man
}

\author{
J OHN A. CLARKE \\ From the Department of Anatomy, University of Glasgow
}

\begin{abstract}
Little reference could be found in the literature to the vasa vasorum in the root of neck arteries in man. The main paper is by Lowenberg and Shumacker (1948), who demonstrated the differences in vascular patterns between arterial and venous vasa in canine carotid arteries by staining red cells with benzidene and concluded that this method would be suitable for investigating the role of the vasa vasorum in arterial repair and transplantation.
\end{abstract}

An account of the examination of the vascular patterns and distribution in the wall of the human root of neck arteries will be given, using the Coslett-Nixon $x$-ray projection microscope. This gives an opportunity of examining the vasa vasorum in full thickness arterial wall without histological preparation, in contrast to the routine injection, clearing, and histological techniques for examining capillary beds by previous investigators (Pickworth, 1934 ; O’Neill, 1947 ; Williams, 1948 ; Scharrer, 1950 ; and Woerner, 1959).

\section{MATERIAL AND METHODS}

Fifty normal aortic arches with the attached root of neck arteries were obtained within 12 hours of death and examined in equally divided five-year groups between the fifteenth and eightieth years.

The vasa vasorum were demonstrated by injecting micropaque (particulate diameter $0.5 \mu$ or less) at manometrically controlled physiological pressures. The arterial vasa were shown by injecting through the brachiocephalic trunk and bronchial arteries, the venous vasa through the bronchial veins.

$X$-ray projection micrographs of full thickness artery wall and $1 \mathrm{~mm}$. thick sections, cut by hand, were taken on Ilford contrasty plates with an exposure time of 10 minutes. The microscope was operated at $15 \mathrm{kV}$ and $40 \mathrm{~mA}$ with a copper target providing the $x$-radiation.

When comparing the vascular densities of specimens, the vessels were estimated against a radioopaque mesh (200 squares/inch), which was superimposed on the specimen when the micrograph was taken. The vessels in each square were then counted, the density measurement being the number of vessels over the total number of squares.

The intrinsic vascular arrangements were studied histologically in 10 specimens by Pickworth's (1934) method (sodium nitroprusside and benzidene) and routine preparation and staining techniques with Mallory's trichrome stain, to provide controls and compare with the results of $x$-ray microscopy.

\section{RESULTS}

RADIOLOGICAL OBSERVATIONS It was evident from the micrographs that the proximal arterial supply to the root of neck arteries originated from the arteriolar plexus on the summit of the aortic arch, the vascular patterns of the latter having been described in a previous communication (Clarke, 1965).

The arterial vasa consisted of parallel, longitudinally arranged, coiled, adventitial arterioles, 80 to $100 \mu$ in diameter, which surrounded the brachiocephalic trunk, left common carotid and left subclavian arteries (Figs 1 to 3 ). In the brachiocephalic trunk and left subclavian arteries the arterioles anastomosed distally with arterial vasa originating from the thyrocervical trunk and internal mammary arteries (Fig. 4).

From the adventitial arterial network, arterial vasa penetrated the outer third of the media to form a secondary plexus of vessels 10 to $20 \mu$ in diameter (Fig. 5).

From an examination of the micrographs it was concluded that the venous vasa originated in the middle third of the media, traversed the walls of the root of neck arteries, and drained into longitudinally arranged veins, 80 to $100 \mu$ in diameter, which were tributaries of the aortic arch venous plexus (Figs 6 to 8 ).

HISTOLOGICAL OBSERVATIONS In general the histological observations on the vasa vasorum confirmed the previous descriptions for the root of neck arteries. All the specimens that were 


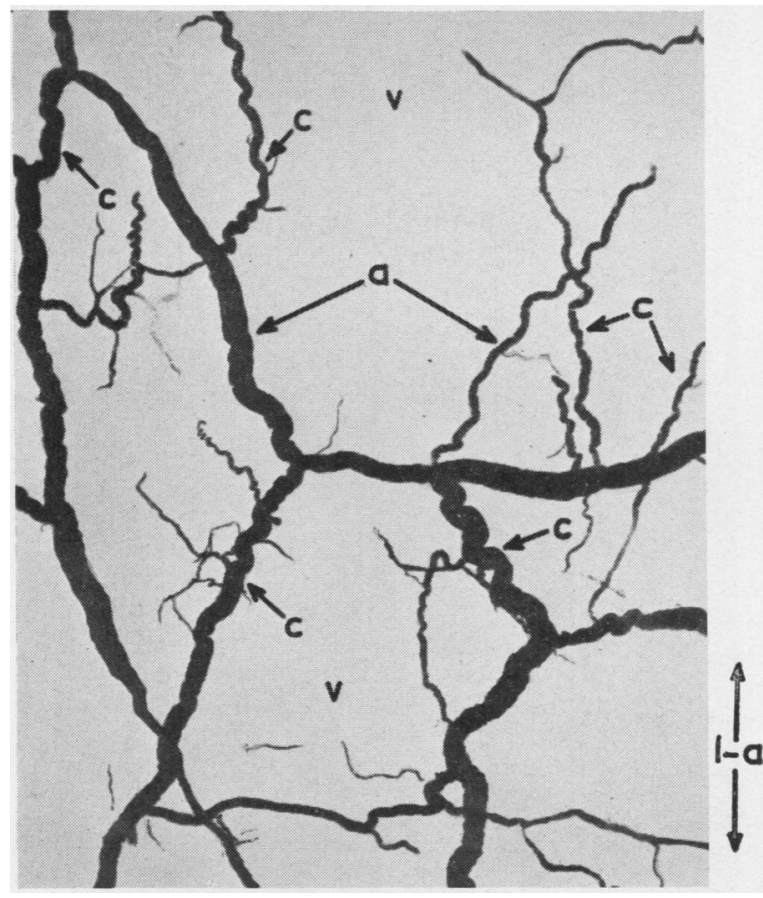

FIG. 1

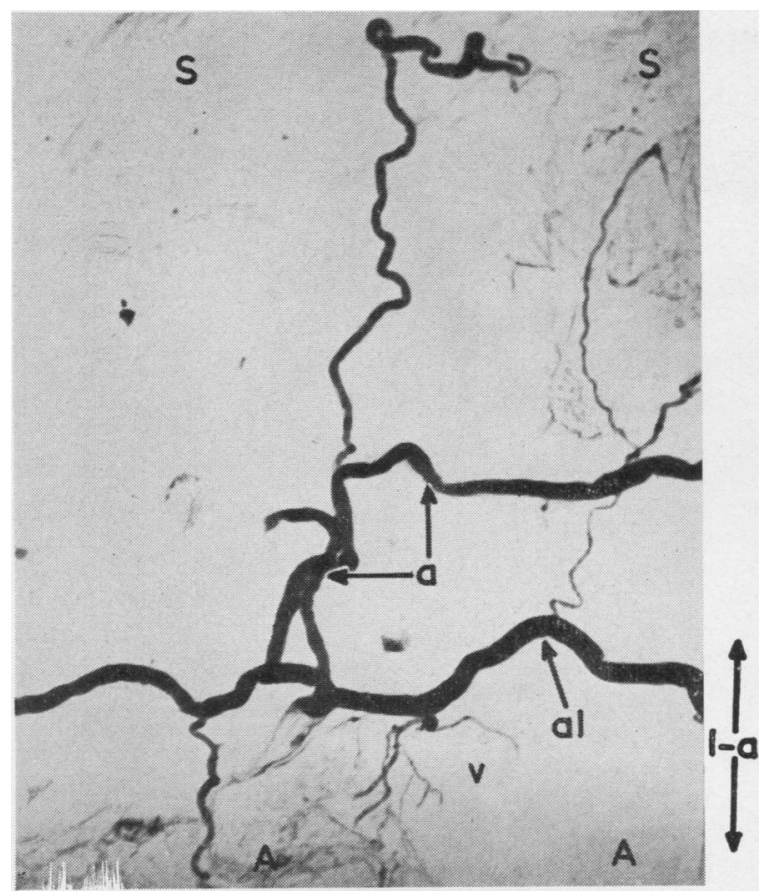

FIG. 3

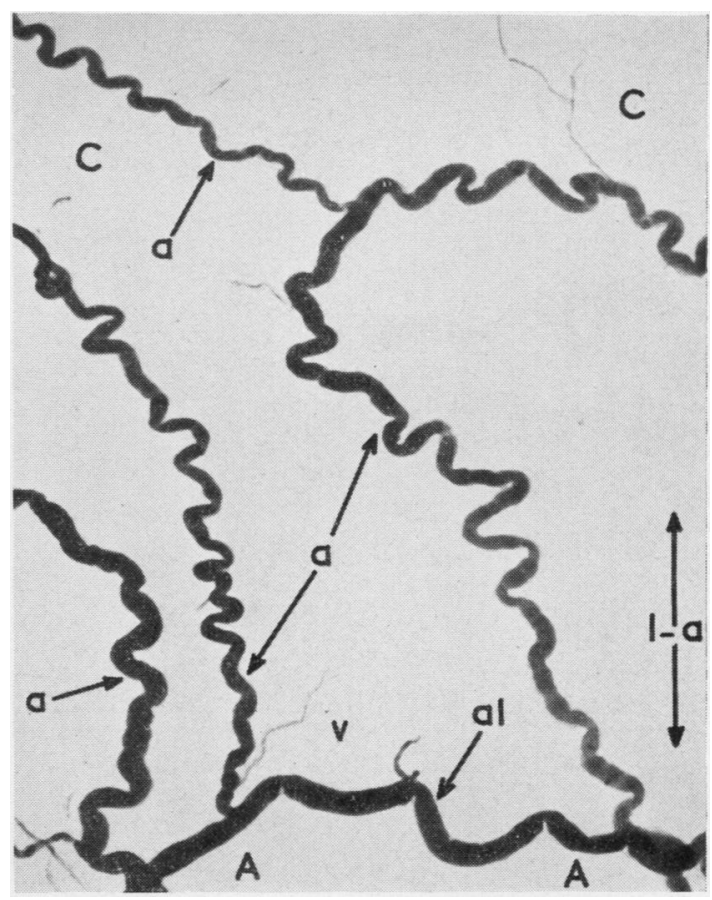

FIG. 2

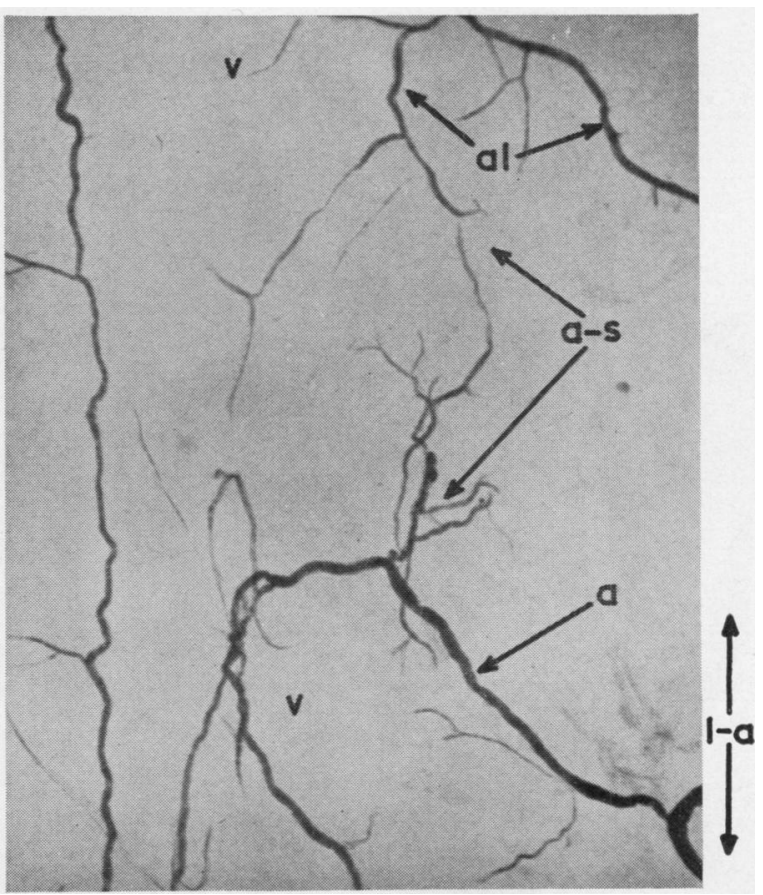

FIG. 4 


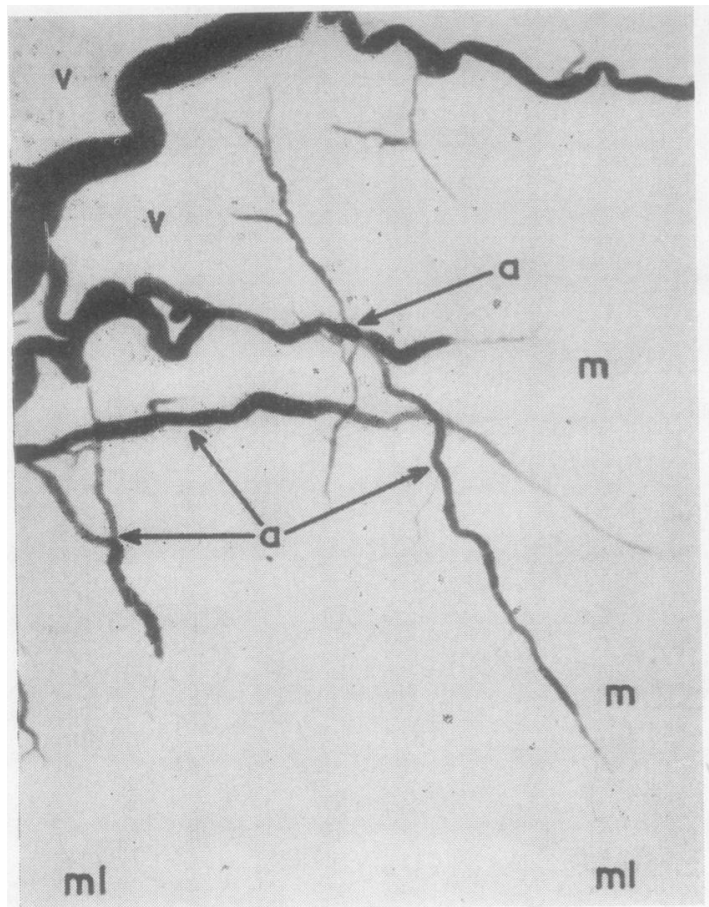

FIG. 5

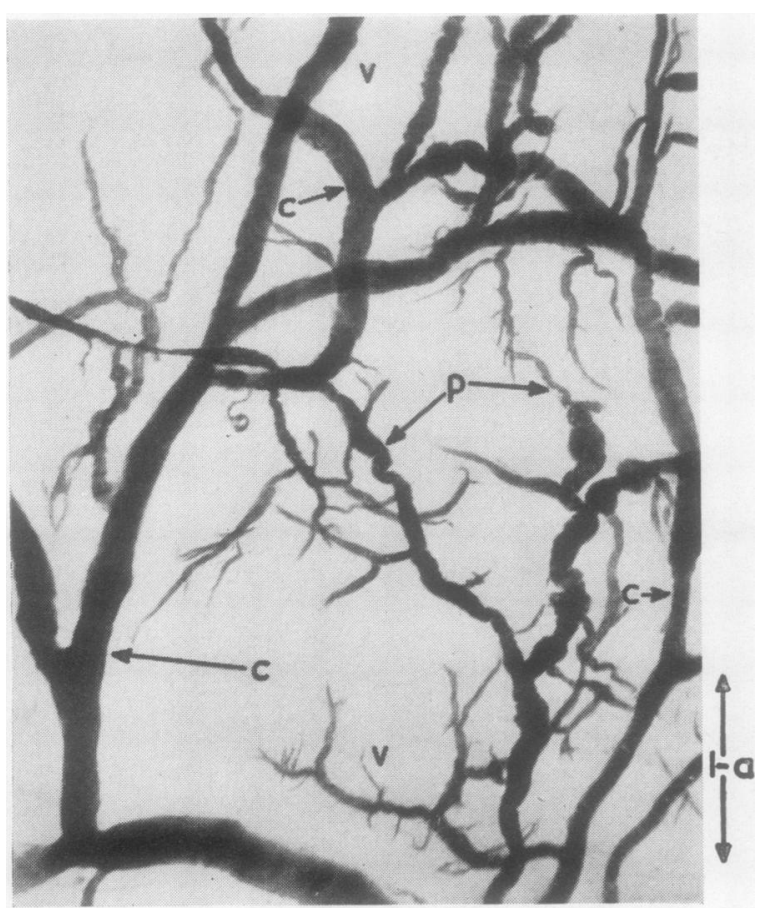

FIG. 7

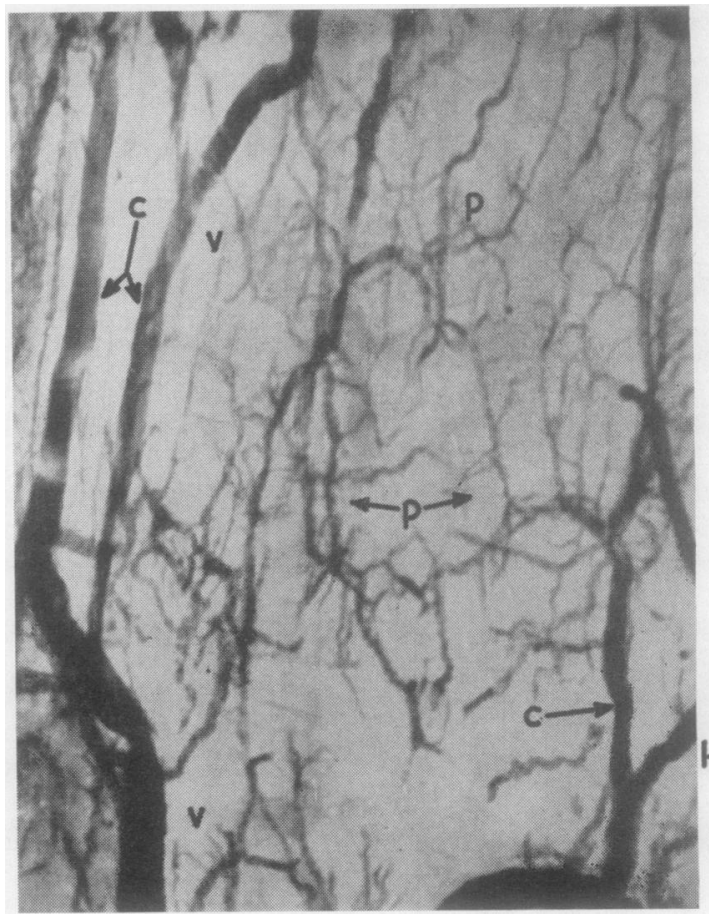

FIG. 6

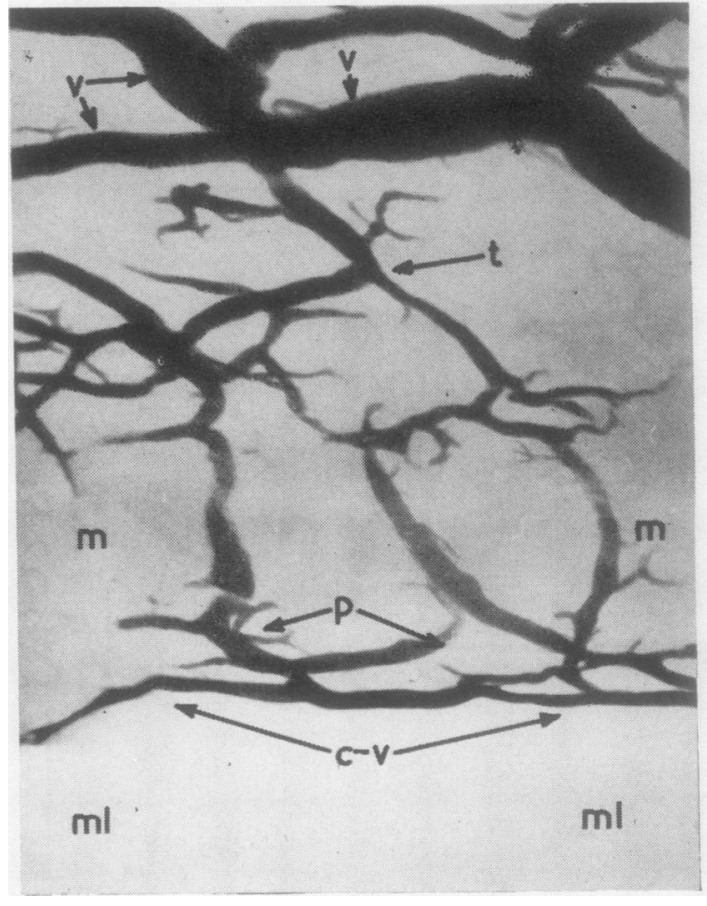

FIG. 8 


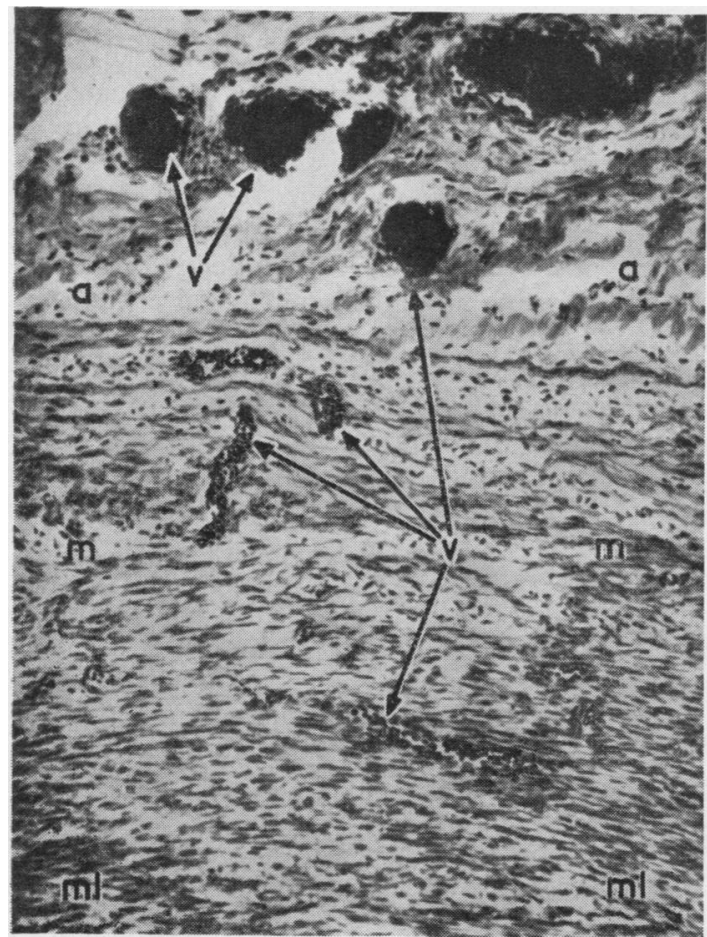

FIG. 9

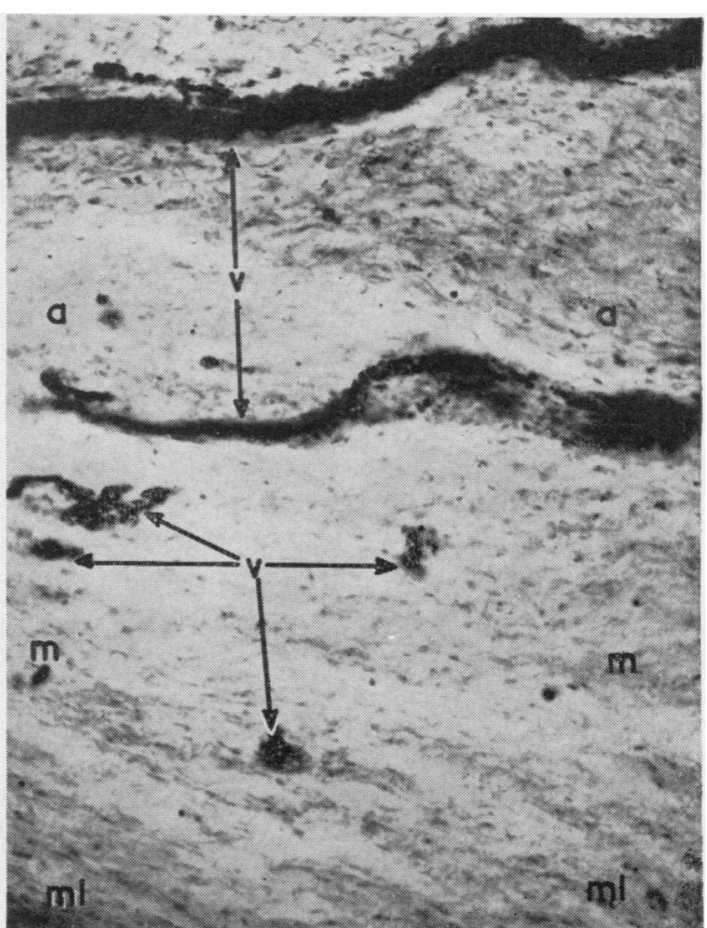

FIG. 10

\section{EXPLANATION OF PLATES}

All micrographs are of full thickness artery wall, except Figs 5 and 8. Long axis of artery (1-a).

FIG. 1. Micrograph of the brachiocephalic trunk showing the arterioles $(a)$ in the adventitia $(v)$. Note coiling $(c), \times 40$.

FIG. 2. Micrograph of the left common carotid artery $(C)$ and the aortic arch $(A)$. Note the coiled arterioles $(a)$ originating from an arteriole $(a l)$ in the adventitia $(v), \times 40$.

FIG. 3. Micrograph of the left subclavian artery $(S)$ and the aortic arch $(A)$. Note the arteriole $(a)$ originating from an arteriole (al) in the adventitia (v) of the arch. Note less dense distribution of arterial vasa. Compare Figs 1 and 2, $\times 40$.

FIG. 4. Micrograph of the brachiocephalic bifurcation. Note the anastomoses $(a-s)$ between the proximal arteriole (a) from the aortic arch and the arteriole (al) from the thyrocervical trunk. Adventitia (v), $\times 20$.

FIG. 5. Micrograph of $1 \mathrm{~mm}$. thick transverse section of the brachiocephalic trunk. Note the arterioles (a)penetrating and supplying the outer third of the media (m). Middle third of the media $(\mathrm{ml})$ is avascular. Adventitia $(v), \times 100$.

f1G. 6. Micrograph of the brachiccephalic trunk. Note the venous plexus $(p)$ in the adventitia (v) draining into longitudinal channels $(c), \times 50$.

FIG. 7. Micrograph of the left subclavian artery. Note the venous plexus $(p)$ in the adventitia (v) draining into longitudinal channels $(c)$. Compare with Fig. 6 for vascular density, $\times 50$.

FIG. 8. Micrograph of $1 \mathrm{~mm}$. thick longitudinal section of the left common carotid artery. Note the origin of the venous plexus $(p)$ in the middle third of the media $(m)$ draining into tributaries $(t)$ of the adventitial veins (v). Capillaryvenule bed $(\mathrm{c}-\mathrm{v})$. Inner third of media $(\mathrm{ml})$ is avascular, $\times 110$.

FIG. 9. Photomicrograph of $8 \mu$ transverse section of the left subclavian artery. Note the vasa vasorum (v) in the adventitia $(a)$ and outer media $(\mathrm{m})$. Inner media $(\mathrm{ml})$ is avascular. Mallory, $\times 200$.

FIG. 10. Photomicrograph of $50 \mu$ longitudinal section of the brachiocephalic trunk. Note the vasa vasorum $(v)$ in the adventitia (a) and outer media $(\mathrm{m})$. Inner media $(\mathrm{ml})$ is avascular. Sodium nitroprusside and benzidene, $\times 150$. 
examined showed vasa in the adventitia and outer media, but the arterial and venous vasa could not be differentiated (Figs 9 and 10).

\section{DISCUSSION}

The present study has shown that there is an extensive vascular supply to the root of neck arteries, the general features of which are shown in Figure 11.

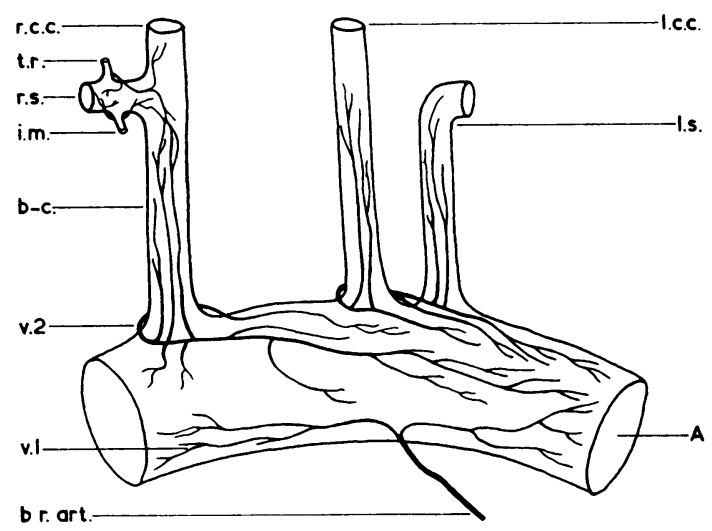

FIG. 11. Diagram of the arterial supply to the root of neck arteries from the aortic arch $(A)$ and thyrocervical trunk (t.r.) and internal mammary artery (i.m.). Note vasa (v.l) originating from a terminal branch of the brcnchial artery (br. art.) and vasa (v.2) from the brachiocephalic trunk (b-c.). Left common carotid artery (l.c.c.): left subclavian artery (l.s.): right common carotid artery (r.c.c.): right subclavian artery (r.s.).

The arteriolar plexus on the brachiosephalic trunk was the richest and most markedly coiled distribution of the three root of neck arteries, and the arterial supply to the subclavian artery was the poorest (Figs 1 to 3 ). The coiling of the adventitial arterioles was regarded as a defence mechanism against the stretch effect of systole.

The arterial supply to the bifurcation of the brachiocephalic trunk from the thyrocervical trunk and internal mammary artery of the right subclavian anastomosed freely with the proximal arteriolar channels from the summit of the aortic arch. A similar anastomosis occurred on the left subclavian artery at the level of the thoracic inlet.

The longitudinal venous plexus, richest on the brachiocephalic trunk and poorest on the left subclavian, was similar in arrangement to the adventitial veins described on the ascending aorta (Clarke, 1964).

In this work arterial and venous vasa could be differentiated in vessels with a diameter greater than $20 \mu$.

Since recognizable arterioles, $20 \mu$ in diameter, could be identified in the outer third of the media. and vessels 25 to $30 \mu$ in diameter could be differentiated in the middle third of the media. only after the venous side of the microcirculation had been injected, it was concluded that the capillary-venule bed lay in the middle third of the media (Fig. 8).

With age no differences in the pattern or distribution of the vasa could be seen.

In contrast to injection methods, techniques which require the staining of red cells depend on the uniform filling of the capillaries at the time of death, and failure to demonstrate vessels by Pickworth's method may be due to lack of blood within the mural vessels. Demonstration of vasa vasorum by routine staining techniques or by showing alkaline phosphatase in endothelial cells (Scharrer, 1950) does not allow the pattern of the mural vessels to be appreciated, in contrast to the routine $x$-ray micrograph.

\section{SUMMARY}

The arterial and venous vasa of normal human root of neck arteries have been shown.

The arterial vasa originated from the arteriolar plexus on the aortic arch proximally, and distally, in the case of the brachiocephalic trunk and left subclavian artery, from the thyrocervical trunk and internal mammary arteries.

The venous vasa formed longitudinally arranged plexuses which drained into the aortic arch venous plexus.

The capillary-venule bed lay in the middle third of the media.

No injection medium entered the inner third of the media or intima.

\section{REFERENCES}

Clarke, J.A.(1964). An $x$-ray microscopic study of the vasa vasorum of the normal human ascending aorta. Brit. Heart J., 27, 99.

(1965). An $x$-ray microscopic study of the vasa vasorum of the normal human aortic arch. Thorax, 20,76.

Lowenberg, R. I., and Shumacker, H. B., Jr. (1948). Experimental studies in vascular repair. III. Morphologic observations of ntudies in vascular repair. III. Morphologic obser

O'Neill, J. F. (1947). The effects on venous endothelium of alterations in blood flow through the vessels in vein walls, and the possible relation to thrombosis. Ann. Surg., 126, 270.

Pickworth, F. A. (1934). A new method of study of the brain capillaries and its application to the regional localisation of menta disorder. J. Anat. (Lond.), 69, 62 .

Scharrer, E. (1950). A technique for the demonstration of the blood vessels in the developing central nervous system. Anat. Rec., $107,319$.

Williams, T. W. (1948). The visualization of vertebrate capillary beds by intravascular precipitation of lead chromate. Ibid., 100, 115. Woerner, C. A. (1959). Vasa vasorum of arteries, their demonstration and distribution. In The Arterial Wall, ed. A. I. Lansing, pp. 1-14. Williams and Wilkins, Baltimore. 